\title{
Global Steering of Single Gimballed Control Moment Gyroscopes Using a Directed Search ${ }^{\dagger}$
}

\author{
Joseph A. Paradiso*
}

The Charles Stark Draper Laboratory, Inc. Cambridge, MA. 02139

\begin{abstract}
A guided depth-first search that manages null motion about torque-producing trajectories calculated with a singularity-robust inverse is proposed as a practical feedforward steering law that can globally avoid (or minimize the impact of) singular states in minimally-redundant systems of single gimballed control moment gyroscopes. Cost and heuristic functions are defined to guide the search procedure in improving gimbal trajectories. On-orbit implementation of the steering law is proposed as an extension to momentum management algorithms. A set of simulation examples is presented, illustrating the search performance for a minimally-redundant, pyramid-mounted array. Sensitivities of feedforward gimbal trajectories are examined in the presence of unmodelled disturbances, and techniques are proposed for avoiding excessive divergence.
\end{abstract}

\section{Introduction}

The next generations of manned and unmanned spacecraft will require enhanced control algorithms in order to efficiently achieve their proposed mission objectives. Regularly coping with uncertainties in the orbital environment, dynamically changing spacecraft configurations (i.e. via docking and buildup), nonlinear actuator properties, and the need to tolerate potential hardware failures will mandate development of control strategies considerably beyond the available state-ofthe-art. Because of the priority placed on minimizing cost, weight, and consumable requirements, future spacecraft will not always be able to rely on highly complex, multiply-redundant actuator systems (as is often now the case), but must employ more flexible and intelligent schemes that efficiently exploit all available onboard control capability.

† Presented at the 1991 AIAA Guidance and Control Conf., New Orleans, LA as Paper 91-2718

* Senior Technical Staff, Member AIAA 
This study has examined methods of applying heuristic search techniques to perform adaptable inverse kinematics and actuator management for spacecraft attitude control. In particular, Single Gimballed Control Moment Gyros (SGCMGs) were chosen as torque actuators. While many existing algorithms may suffice for steering Double Gimballed CMG (DGCMG) arrays, such as envisioned for the NASA Space Station, no powerful techniques have been developed to manage minimally redundant arrays of SGCMGs. These devices, however, are ideally suited as momentum-exchange effectors for a wide class of future spacecraft because of their large torque output and momentum capacity. They offer significant cost, power, weight, and reliability advantages over DGCMGs and could provide attitude control for a wide variety of future spacecraft, as torque requirements in many proposed moderate-sized vehicles may surpass the capability of available reaction wheels. Due to the complicated nonlinear mapping between the input (gimbal) space and output (momentum) space, however, effective steering laws that reliably avoid problematic singular states have not been developed for minimally-redundant SGCMG systems, discouraging their application in many situations. In order to fully exploit the capability of SGCMGs, intelligent steering laws must be developed that address the system nonlinearities and avoid singular states over global trajectories.

This effort has produced an effective steering algorithm for SGCMGs using search techniques commonly applied in solving trajectory optimization problems. Employing a momentum profile assumed to be forecast by a momentum management procedure (or maneuver scheduler), the search-based steering law generates a set of feedforward gimbal trajectories that avoid singular configurations (or minimize the effect of any singularities that are unable to be avoided), while maintaining hard constraints on gimbal rates. While the CMGs follow the prescribed trajectories, a "watchdog" regulator task nulls local disturbances and monitors the divergence of gimbal trajectories from their projected values. By using a global planning algorithm of this sort as a feedforward CMG steering law, one is effectively uniting many aspects of the CMG steering process with a momentum management algorithm. 
In the orbital environment, the feedforward gimbal trajectories produced by the search process will be realized in the presence of unmodelled disturbances. The susceptibility of gimbal trajectories to unknown secular torques is examined in this report. In certain cases, particularly those with CMGs approaching the vicinity of a singular state, significant divergence from the feedforward path can occur, rapidly increasing after the singular encounter. Suggestions are proposed for dealing with this situation.

\section{CMG Kinematics and Steering}

Control Moment Gyros (CMGs) are momentum exchange actuators that consist of a constant-rate flywheel mounted on a gimbal (or set of gimbals). By torquing the gimbal(s), the flywheel orientation is changed, thereby re-directing the rotor's angular momentum. The net momentum stored in a CMG array onboard a spacecraft is equal to the vector sum of all CMG rotor momenta. As individual gimbal angles are varied, the net $\mathrm{CMG}$ momentum may be continuously and deliberately adjusted. In accordance with conservation of angular momentum, any change in stored CMG momentum must be transferred to the host spacecraft, producing a reaction torque.

CMGs are available as single (SGCMG) or double (DGCMG) gimballed devices. From the hardware viewpoint, the SGCMG is the simplest (Fig. 1a). The rotor is constrained to rotate on a circle in a plane normal to the gimbal axis $\hat{\sigma}$, hence the orientation of its angular momentum vector is specified through the gimbal angle $\theta$. Because of the restricted freedom per device (i.e. torque and momentum constrained to a fixed plane), optimal inverse kinematics (termed "CMG steering") can be extremely complicated.

DGCMGs are considerably easier to use. The rotor is suspended inside two gimbals, hence the rotor momentum can be oriented on a sphere, along any direction (assuming no restrictive gimbal stops). Double gimballed CMGs are generally significantly heavier, consume more power, are considerably more complicated (thus potentially less reliable), and appreciably more expensive than single gimballed devices. The steering problem is much simpler, however; because of the extra 
degree of freedom per device, DGCMGs can be easily managed by simple steering laws exploiting specific mounting protocols[1], pseudoinverse approaches[2], or linear programming[3]. A standard application of DGCMGs has been in the momentum management of large spacecraft, where a set of flywheels carrying copious momentum are lazily gimballed about, nulling small environmental torques over each orbit and cyclically exchanging significant amounts of angular momentum. DGCMGs were used to control Skylab attitude[4] and are proposed for use onboard the planned NASA space station[5].

Because of their myriad hardware advantages, consideration of SGCMGs has begun to enter into traditional DGCMG applications; i.e. attitude control of large spacecraft, as in the Soviet MIR space station, stabilized by a 6 SGCMG array[6]. Since current steering laws experience difficulty avoiding singularities, these SGCMG systems must be grossly oversized to place all unescapable singular states outside of the required momentum reservoir, leading to considerably increased expense.

Reaction wheels are conventionally used to control the attitude of 3-axis stabilized satellites and smaller spacecraft. These devices vary the spin of a fixed-axis flywheel to transfer momentum and directly couple torque to a host vehicle. Although they are the simplest, lightest, and least expensive of all momentum-exchange effectors, they achieve lower bandwidth and much smaller torque capability than CMGs (no amplification principle is at work; torques are coupled directly into the spacecraft). As larger spacecraft and satellites are constructed, SGCMGs may prove to be a necessary and cost-effective upgrade from reaction wheel systems. Before SGCMG arrays can compete efficiently in any of these arenas, however, a general steering algorithm must be developed that can successfully manage a minimally-redundant SGCMG array.

Fig. 1 shows a drawing of a basic SGCMG device. Moving a CMG rotor about its gimbal axis produces a precession torque on the spacecraft:

1)

$$
\underline{\tau}_{\mathrm{CMG}}=-(\hat{\sigma} \mathbf{x} \underline{\mathrm{h}}) \theta \quad \theta=\mathrm{CMG} \text { Gimbal angle }
$$


The control variables for a system of N CMGs are the gimbal rates $\dot{\theta}_{i}$, which are adjusted to yield a commanded torque. One may thus state the CMG control constraint:

$$
\underline{\mathbf{\tau}}_{\mathrm{cmd}}=[\mathbf{J}(\underline{\theta})] \underline{\theta}
$$

The matrix $\mathbf{J}(\underline{\theta})$ is the $(3 \times \mathbf{N})$ Jacobian of the CMG system's total momentum with respect to the gimbal angles. Its columns are the individual CMG output torque authorities, $\underline{\tau}_{i}=\hat{\sigma}_{i} \times \underline{h}_{i}$. The CMG steering problem is effectively an inversion of Eq. 2; namely specify a set of gimbal rates that deliver $\underline{\tau}_{\mathrm{cmd}}$ while meeting constraints (i.e. hardware limits on maximum gimbal rates) and managing CMG system redundancy in an "optimal" fashion, such that singular gimbal states and undesirable rotor \& gimbal orientations are avoided.

For most relevant applications, the CMG array will be required to maintain full 3-axis attitude control. Situations exist, however, where the CMG rotors are configured such that the Jacobian in Eq. 2 loses rank, and control authority can not be projected along a particular axis. Such gimbal configurations, termed singular states, are clearly undesirable.

The simplest singular state is momentum saturation, defined as the "momentum envelope". This is a 3-dimensional surface that represents the maximum angular momentum attainable by the CMG array along any given direction. It is analogous to the "workspace" boundary of the robotic manipulator[7] (i.e. the surface of maximum reach). The saturation state is impossible to avoid through exploiting the excess degrees of CMG freedom. It must be directly addressed by the momentum management scheme. Fig. 2a shows a calculation[9] of the momentum envelope for a 4-SGCMG pyramid mounted system (Fig. 6). The envelope is roughly spherical, marred by concave "dimples" along the directions of the gimbal axes.

Other singular configurations exist, however, with net momenta inside the envelope. These are characterized by a combination of maximal and minimal rotor projections onto a given singular direction (in general, there are $2^{\mathrm{N}}$ possible singular states for an N-rotor system along any arbitrary axis[8]). A CMG steering law (or inverse kinematic procedure) manages system redundancy to 
avoid internal singularities while answering torque commands. Fig. $2 b$ shows a cutaway view of the envelope and internal singular states. The geometric complexity of the singularities is immediately evident, and is discussed in detail in Refs. $[9,10]$. The convoluted nature of these singular surfaces prevents their simple anticipation and avoidance in steering laws (the singular surfaces of an ideal DGCMG array, in contrast, are purely spherical).

By taking the determinant of the square matrix formed when multiplying the Jacobian by its transpose, one obtains a quantity (referred to here as the CMG gain[11]) that reflects the Jacobian's rank. The square root is conventional in most formulations.

$$
\mathrm{m}=\sqrt{\left|\mathbf{J J}^{\mathrm{T}}\right|}
$$

In the present context, $\left[\mathbf{J} \mathbf{J}^{\mathrm{T}}\right]$ is a $3 \times 3$ matrix. As $\mathrm{m}$ approaches zero, the system is nearly singular, and an axis of control is effectively lost.

Singular states fall into two categories[8]; "hyperbolic" states that can be escaped through null motion (i.e. redistributing the $\mathrm{CMG}$ rotors to relieve the singularity without changing the momentum state of the spacecraft), and "elliptic" states that are inescapable through null motion. The latter "inescapable" states pose a major difficulty with SGCMG systems. They arise from the limited control capability per actuator; i.e. using a collection of single DOF devices to control a 3axis space. With devices such as DGCMGs (2 DOFs per actuator), inescapable singular states are either nonexistent or trivial (i.e. the envelope of any system is technically a "trivial" inescapable singularity). Depending upon the direction of the torque command and the initial gimbal state, certain singular states of SGCMG systems may be unavoidable[12], and must be transited.

Analysis techniques have been applied[8,13] to classify the internal singular states of the pyramid-mounted 4-SGCMG system. The outermost surfaces (with one rotor anti-aligned) were generally found to possess an elliptic (inescapable) character, while the innermost surfaces (with opposing pairs of rotors) were generally seen to be hyperbolic (escapable). Since they can not always be relieved without torquing the spacecraft, the outermost singularities can be especially 
problematic for SGCMG systems and steering laws. Proposed SGCMG deployments generally size the CMG array to keep the required momentum capacity within the boundary of these surfaces. This considerably reduces the available momentum (the effective reduction can be gleaned from Fig. 2). In order to operate in the regions of inescapable singularities, an intelligent steering law must be developed that avoids elliptical states where possible, or rapidly transits elliptical configurations in momentum space, while minimizing their effects on the spacecraft momentum state and attitude control.

The purpose of a "conventional" CMG steering law is to determine CMG gimbal rates that answer instantaneous torque commands, while managing the system redundancy to maintain desirable (i.e. nonsingular) gimbal states. Most CMG steering laws calculate torque-producing gimbal rates with some variant of pseudoinverse.

4)

$$
\underline{\dot{\theta}}_{\tau}=\mathbf{J}^{\mathrm{T}}\left(\mathbf{J J}^{\mathrm{T}}\right)^{-1} \underline{\boldsymbol{\tau}}_{\mathrm{cmd}}
$$

The pseudoinverse produces the minimum 2-norm vector of gimbal rates $\underline{\dot{\theta}}_{\tau}$ that realize the commanded torque $\underline{\tau}_{\text {cmd }}$. If these gimbal commands are applied to a CMG system without modification, however, the minimum norm property of the pseudoinverse encourages the formation of singular states.

The pseudoinverse can be considered as a "particular" solution to the torque equation, Eq. 2 . The corresponding homogeneous solution is produced through null motion, which describes gimbal motion that does not change the CMG momentum state and torque the spacecraft. Null motion may be calculated in a variety of manners; i.e. through a singular value decomposition[14], a projection operator[15], or a cross product[8].

The particular and homogeneous solutions are summed to form a general relation that spans all possible $\mathrm{CMG}$ motions that attain the commanded torque:

$$
\underline{\dot{\theta}}_{\mathrm{cmd}}=\underline{\dot{\theta}}_{\tau}+\sum_{1=1}^{\mathrm{N}} R_{\mathrm{i}} \underline{\theta}_{\mathrm{N}_{(\mathrm{i})}}
$$


The CMG steering law assigns weights $R_{\mathrm{i}}$ to the system's null vectors $\underline{\theta}_{\mathrm{N}_{(i)}}$ to determine gimbal motion that avoids singular and problematic states. For the 4-CMG system, this amounts to picking a signed scalar variable, since there is only one null vector. Most (if not all) steering laws are local in nature, hence solve Eq. 5 for gimbal rates that are instantaneously "optimal" at the current gimbal configuration. The $R_{i}$ factors are thus picked at each time step. An objective function can be defined to reflect desirable gimbal orientations, and the $R_{\mathrm{i}}$ factors can be calculated by differentiating with respect to null vector displacement.

Although such "gradient" techniques can work well with DGCMGs[2], they generally have considerable difficulty in managing SGCMG systems of limited redundancy. Steering laws that locally track the maximum of an objective function tend to often be drawn into the complicated mire of singular states.

In order to avoid this difficulty, a variety of analytic and heuristic approaches have been developed to determine a singularity-avoiding null motion policy. These have involved several local gradient procedures[7], singularity ranking and rotor "unkinking" strategies[15], lookup table consultation[11], assignment of particular initial gimbal angles[16], and configuration-based techniques[6]. In general, existing methods either exhibit unreliable singularity avoidance, or appreciably constrain the operation of SGCMG systems. In order to improve performance, the ideal steering algorithm must be "global" in nature, and optimize the CMG gimbal motion over a predicted momentum trajectory.

\section{Global CMG Steering Using a Guided Search}

A guided depth-first search has been adapted to optimize CMG gimbal trajectories between "goal" states specified by a momentum manager or spacecraft maneuver scheduler. The search uses a Singularity-Robust (SR) inverse to calculate gimbal rates that achieve a commanded torque scheduled by the momentum manager or anticipated maneuver. The SR inverse is a modification to the pseudoinverse that has been investigated for CMG application in Ref. [7]. When the CMG 
gain begins to decrease, and the system goes singular, the SR technique adds a perturbation to the $3 \times 3 \mathbf{J J}^{\mathrm{T}}$ matrix in order to artificially maintain rank and retain invertibility.

$$
\underline{\dot{\theta}}_{\tau}=\mathbf{J}^{\mathrm{T}}\left(\mathbf{J} \mathbf{J}^{\mathrm{T}}+\rho \mathbf{I}_{3}\right)^{-1} \underline{\boldsymbol{\tau}}_{\mathrm{cmd}}
$$

Eq. 6 is identical to the pseudoinverse (Eq. 4), except for the term proportional to the $3 \times 3$ identity matrix $\mathbf{I}_{3}$. The constant of proportion, $\rho$, is made to be negligible away from a singular state, and increase rapidly as the singularity is approached. The effect of the SR inverse near a singular state is to drop the rank of the torque constraint (Eq. 5); gimbal rates are not generated to answer command components about the singular axis. Although this can produce significant momentum error[7], the SR inverse potentially allows the CMG configuration to transit a singular state without specifying unphysically large gimbal rates. The search is executed to calculate a null motion function $[R(t)]$ that avoids singularities where possible, and minimizes the SR-induced errors where a singularity is unavoidable (the SR-inverse gives the search the ability to specify gimbal trajectories that cross singularities, in exchange for possible momentum error).

The coordinates used in the search are outlined in Fig. 3a. The horizontal component is the predicted torque command, specified at several discrete intervals. The search varies the added null motion at each such point to evade singular states (or minimize the time spent in an unavoidable singularity and its consequent momentum error).

The amount of null motion added at each point (to generate the successor) is also discretized to form a tree structure that starts at an initial node representing the CMG configuration expected at the beginning of an orbit or maneuver. The tree proceeds, branching at every interim point where an intermediate momentum state is specified, and ending at a range of possible terminal states that exhibit the final commanded net CMG momentum. The density of nodes at the terminal state can be extremely high (i.e. the number of null motion "children" attached to a parent node raised to the power of the number of momentum steps), thus arriving at an optimal solution to this problem can be quite difficult. Fortunately, an optimal solution is unnecessary; any solution that 
avoids a singularity, or quickly crosses it without large ill-effect, may satisfice our requirement. Heuristic functions and node-pruning can thus be introduced that enable an adequate solution to be rapidly located without exploring (i.e., "opening") comparatively many nodes.

The set of child nodes expanded from a parent spans the range of possible null motion, as depicted in Fig. 3b for a 4-CMG system (scalar R). The center child represents gimbal motion performed between the two timesteps without adding null motion; i.e. $k=0$ in Eq. 6 , and the SR inverse solution is applied without modification. The nodes extending above (and below) the center denote transitions between parent and child momentum states that introduce positive (and negative) null motion via a signed value of $R$. The nodes are generally equally spaced in $k$, and the range in $R$ spanned between upper and lower nodes is normalized such that the node corresponding to maximum (or minimum) null motion runs the gimbals to within a preset fraction $(\eta)$ of their peak hardware rate limits. The dynamic range in added null motion accessible to the search is thus set by the number of child nodes per parent.

As more CMGs are added, the dimension of the nullspace is likewise incremented, and the problem can become much more complicated; i.e. the amount of nodes (hence storage) needed can rise with the power of the search dimension, which is equal to the number of null degrees of freedom. Fortunately, the SGCMG kinematics again allow a loophole out of this situation. Singularities become much easier to avoid as more CMGs are added[17]. Even simple gradient steering techniques begin to function adequately with 5 or more SGCMGs in a system. The search process may not need to work very hard to improve a gradient solution in a larger CMG system, thus the search can be constrained without sacrificing performance, as suggested in Ref. [9].

The search process selectively "expands" promising nodes by "opening" all of their children (i.e. making them available to candidate gimbal trajectories). Each node expanded by the search represents a specific CMG gimbal orientation. A numerical "cost" can be associated with each node to express the optimality of the corresponding gimbal position, as quantified below:

$$
\mathrm{c}_{\mathrm{n}}=\mathrm{W}_{1}\left(m_{\min }\right)_{\mathrm{n}}^{2}-\mathrm{W}_{2} \sum_{\mathrm{j}=1}^{\mathrm{n}}\left(\frac{1}{m_{\mathrm{j}}}\right)^{2}-\mathrm{W}_{3} \sum_{\mathrm{j}=1}^{\mathrm{n}}\left|\underline{\mathrm{h}}_{\text {res }(\mathrm{j})}\right|^{2}-\mathrm{W}_{4} \sum_{\mathrm{j}=1}^{\mathrm{n}}\left(\Delta \dot{\theta}_{\text {over }}\right)_{\mathrm{j}}-\mathrm{W}_{5} \sum_{\mathrm{j}=1}^{\mathrm{n}} \mathrm{J}_{\text {null }}(\mathrm{j})
$$




\section{Where:}

$m_{\mathrm{j}}=\mathrm{CMG}$ gain (re. Eq. 3) at node $\mathrm{j}$.

$m_{\min }=$ Minimum value of CMG gain over path through node $\mathrm{j}$.

$\underline{\mathrm{h}}_{\text {res(j) }}=$ Momentum residual (commanded vs. delivered) at node $\mathrm{j}$.

$\left(\Delta \theta\right.$ over $_{\mathrm{j}}=$ Net gimbal rate above hardware limit at node $\mathrm{j}$.

$\mathrm{J}_{\text {null }}(\mathrm{j})=\left|k^{(\mathrm{j})}\right|+\left|k^{(\mathrm{j})}-k^{(\mathrm{j}-1)}\right|$

$k^{(j)}=$ Null motion added at node $\mathrm{j}$.

The objective function given in Eq. 7 is defined to be maximized in an optimization; i.e. the search works to maximize the $c_{n}$ of the terminal node. The summations in Eq. 7 add their arguments back along the tree (tracing parents through a linked list) from node $\mathrm{n}$ to the start node. They represent sums over the nodes along the unique path through the tree that terminates at node $\mathrm{n}$, hence are not sums over all expanded nodes. These sums need not be calculated completely anew at every encountered node, but can be recursively updated; i.e. when a new node is expanded, the current value of the summation argument is added to the previous sum stored at its parent node. The various terms in Eq. 12 act to produce specific CMG steering response, and will be summarized individually below; additional detail is given in Ref. [9].

The first term is proportional to the minimum value of $\mathrm{CMG}$ gain over the trajectory through node $\mathrm{n}$ (i.e. $\inf \{m\}$ ). It is generally weighted somewhat heavily, thus the search works extensively to maximize the worst-case gain value, thereby avoiding singular states.

The second term approximates the integral of the inverse gain. Since it's added negatively, the search works to minimize this quantity. It represents the amount of time spent in the vicinity of a singular state; the longer the trajectory lingers at low gain, the higher this penalty becomes. 
The third term is the amount of momentum residual accumulated over the trajectory through node $\mathrm{n}$, hence penalizes nodes attached to trajectories that have been significantly displaced from their commanded momentum state (as in a close approach to a singularity with the SR inverse).

The fourth term acts to prevent nodes from being selected that exhibit CMG gimbals running over their maximum allowed rates. Since its weight is set very high, this term is assigned the utmost importance in the optimality criterion. If all $\mathrm{CMG}$ gimbals are running below peak hardware limits, $\Delta \theta_{\text {over }}$ is zero, and no amplitude is contributed to the objective. As the gimbals surpass their peak limits, $\Delta \theta_{\text {over }}$ is set to the net excess rate, hence the penalty increases the more the rate constraint is violated. The over-rate penalty is also summed recursively across the trajectory, hence represents all excess rates encountered through node $\mathrm{n}$.

The final term in Eq. 12 reduces undesirable side-effects of null motion. By itself, the pseudoinverse solution usually generates a very smooth and clean gimbal rate profile, due to its intrinsic 2-norm minimization. Gimbal rates can increase when adding null motion; with a small torque command, the added null motion can often dominate the solution, especially when the null freedom is discretized to a small number of allowed levels, as it is under the node structure imposed by our search protocol (i.e. Fig. 3b). If it's not needed to skirt a singularity, one would thus prefer adding little or no null motion. This is encouraged by the first term in the expression for $\mathbf{J}_{\text {null }}$ (i.e. the sum of null amplitudes across the trajectory). Even potentially worse than large unneeded null motion is a null policy that often switches the null vector rapidly on and off, or continually ramps it between positive and negative extremes. These solutions will produce appreciable chatter in the system that can vibrate the spacecraft, consume power, and perhaps eventually degrade the CMG hardware. These considerations are addressed by the second term in the expression for $\mathbf{J}_{\text {null }}$, which represents the magnitude of the difference between null motion added at successive nodes of the trajectory (i.e. a discrete time derivative of the null motion factor $B(t)$ ). This term acts to penalize trajectories exhibiting high-frequency gimbal chatter and prefers solutions with more constant, steady gimbal motion. 
An additional term is added to the objective to avoid quick convergence to local minima, as discussed in Ref. [9].

Immediately before the search is actually executed, a series of trial trajectories are calculated using various gradient techniques. These trajectories are propagated locally node-by-node; i.e. at each step, the child node that best exhibits the assigned criteria is chosen for expansion. The trials thus produce an initial population of open nodes representing gradient solutions; these may be "grafted upon" (i.e. they may be chosen for expansion) and improved by the search process. In addition, the winner of the trials produces an initial "best trajectory", providing a cost cutoff with which to judge and prune subsequent search attempts. The first set of trials is straightforward; i.e. the null motion added at each step is kept constant (the number of such trials is equal to the number of children per parent node). The next trial is a straightforward gradient approach; i.e. the best-cost child node is always selected for expansion. The final trial is a discrete version of the "indirect avoidance" method of Ref. [15]. If all child nodes project all their CMG rotors into the hemisphere of the torque command, the best-cost child node is selected for expansion, as in the previous gradient scheme. If, however, child nodes exist with at least one CMG rotor projecting negatively onto the torque request, the node exhibiting gimbal positions that yield the smallest magnitude of net negative rotor projection is selected for expansion. In this fashion, the rotors are "unkinked" and move into the hemisphere of the torque command, thereby disfavoring standard singular CMG states with antiparallel rotor alignments.

After these trial trajectories are completed, the search begins in earnest to modify and improve on them. The best-cost open node is selected for expansion, then it is closed, and its best cost child is in turn selected for expansion. The search continues looking only at immediate progeny in this fashion until either the terminal state is reached or the cost of the current node is less than the cost attained by an already completed trajectory. In the latter case, the current trajectory is abandoned, and all open nodes are again scanned for that with best cost, from which a new trajectory is launched. In the former case, where the terminal state has been attained, the cost of the terminal node (modified by a term that works to maximize the average CMG gain; see Ref. [9]) 
is compared to that of the currently "best" trajectory. If the new trajectory is better, it inherits the "currently best" definition, and its terminal cost is used as the new discriminant to prune node expansion. In this way, the search dynamically learns its cutoff function, which accomplishes the duty of a heuristic in pruning fruitless search paths; i.e. the search doesn't waste resources exploring a path if its cost drops below that of a trajectory that has previously made it to the terminal state. This cost cutoff is initially quite generous after the trial trajectories, but rapidly becomes more discriminating as the search progresses and superior trajectories are propagated to the terminal state.

The guided depth-first search logic discussed above is summarized in the flow chart of Fig. 4. This procedure somewhat resembles a gradient-based null motion CMG steering law with corrective look-back; i.e. local gradient solutions are pursued until they get into trouble, at which point the trajectory is examined, perturbed at a promising point, and again gradient-propagated. Candidate CMG gimbal trajectories are continually produced with this search technique. The initial trajectories can be of fairly poor quality, especially in cases with difficult singular configurations dominating the current gimbal region, but as the search progresses, the quality of the accepted trajectories gradually improves; the cost cutoff, updated after each trajectory is completed, grows more stringent, and the search wastes less time exploring poor-cost paths. The search process can be stopped when the allotted time and/or computation resources are exhausted, or when the terminal trajectory cost improves past a preset threshold. Additional measures to cut search complexity and speed execution are discussed in Ref. [9].

A diagram is given in Fig. 5 to illustrate a possible on-orbit application of the search-based CMG steering approach. A forecast of the anticipated CMG-stored momentum (i.e. a 90-minute orbit-to-orbit prediction) is assumed to be produced by an onboard momentum manager. The CMG search routine is executed to find a feedforward gimbal trajectory that answers this momentum profile. Ideally, the search is only needed to run once per orbit, and more often (as required) if significant unmodelled disturbance torques are present. The search uses its allotted 
time to globally perfect gimbal trajectories; since the depth-first approach is used, the search may be halted at any time, and the best calculated solution put into service.

The outputs of the search are torque command $\underline{\tau}(\mathrm{t})$ and null vector scaling $\mathrm{R}(\mathrm{t})$ histories that are implemented by a simple CMG steering law, running in a standard high-rate real-time loop. This is a tangent steering law (circa Eq. 12), that calculates both the SR-inverse (for $\underline{\dot{\theta}}_{\tau}$ in response to the input torque command), and null vector(s) from the currently measured CMG gimbal state. Its input torque command is formed by compensating the current value of scheduled torque output from the $\mathrm{CMG}$ search and momentum management routines with an estimate of any disturbance via a loop closed around the vehicle dynamics (needed to force the spacecraft to track the commanded state). In the ideal case (without any disturbance), the vehicle accurately follows the prescribed trajectory, and the simple steering law drives the CMGs exactly as anticipated in the CMG search.

The singularity-avoiding output of the CMG search is a discrete time history of the null vector scaling $R(\mathrm{t})$. As the low-level steering law answers the commanded torque via the SRinverse, it also calculates a null motion vector $\underline{\theta}_{N}$ from the estimated gimbal state at each iteration. Instead of weighting the null motion locally, as performed for singularity avoidance in local steering laws, the value of $R(t)$ is taken from the search output (at the current time " $t$ ", and interpolated from the coarser timesteps used in the search). In this fashion, the real-time steering law uses the global search information to manage null motion and evade singular conditions.

The local nature of the steering law (i.e. calculation of torque and null solutions relative to the instantaneous gimbal state, together with feedback around the vehicle dynamics) always drives the system to track the input torque command. The fed-forward null amplitude $R(t)$, however, is calculated by the search, relative to a predicted gimbal angle trajectory. If the actual gimbal trajectory, as managed by the local steering law, diverges appreciably from the predicted path used in the search (i.e. due to unmodelled disturbances), the fed-forward null amplitude no longer has any relevance, and the CMG system will not necessarily produce the anticipated singularity characteristics. Situations of this sort will be detected by the watchdog regulator task, which 
compares the actual gimbal angles with their predictions. If significant divergence is detected, several provisions may be taken, as discussed in Ref. [9], and outlined in the next section.

If one removes the CMG search block from the implementation diagram of Fig. 5, the logic is essentially identical to that of a standard orbital autopilot. The watchdog regulator is still needed, but it now monitors the momentum state of the vehicle and re-queues the momentum manager upon discovering significant divergence from prediction. The logic of Fig. 5 pulls aspects of the CMG steering (i.e. the nonlinear optimization) up to the level of momentum management, where we now calculate projected gimbal trajectories, as well as momentum storage. One can also interpret the momentum manager in Fig. 5 to be a momentum scheduler in the case of maneuvering vehicles, where momentum values would be fed-forward from an anticipated set of vehicle attitude commands.

\section{4) Simulation Results}

In order to gain experience with the application of a directed search to SGCMG steering, a graphical simulation package has been assembled for the Macintosh computer. Three major programs have been written. An initialization routine allows the user to interactively define commanded momentum histories (i.e. as would arise from a momentum manager). Another program performs the CMG search about these momentum commands to locate satisfactory gimbal trajectories, which are analyzed in the final program for sensitivity to unmodelled disturbances. Details on software implementation are given in Ref. [9].

The CMG mounting scheme used in this study is depicted in Fig. 6. It is a conventional "pyramid mount", where four CMGs are constrained to gimbal on the faces of a regular pyramid

(the gimbal axes $\stackrel{\wedge}{\sigma}$ are orthogonal to the pyramid faces). Each face is inclined at $54.7^{\circ}$ to the horizontal (thus gimbal axes are at the complement angle; i.e. $35.3^{\circ}$ ), yielding a momentum envelope that is roughly spherical for a 4-CMG array (Fig. 2a); i.e. the authority along the vertical (z) axis is similar to the authority that can be projected along $\mathrm{x}$ and $\mathrm{y}$. The mimimally-redundant 4SGCMG configuration encounters the most difficulty with singular states, thus presents a 
significant challenge for CMG steering laws. All examples assume that the CMGs start with initial gimbal angles of zero, although arbitrary initial angles may be specified in the search software. The CMGs are all defined to have unit momentum, and a peak gimbal rate of $1 \mathrm{radian} / \mathrm{sec}$. No gimbal stops are imposed (although they can be directly accommodated in the search software).

The momentum command profile is specified at 30 discrete time-steps, with an assumed time scale of 0.5 second per step (much faster than required for momentum management). The search integrates its gimbal trajectory (i.e. calculates the SR inverse and null motion) twice per step (a linear interpolation is performed to double the number of commanded points). At each step, the momentum residual from the previous integration is added into the new command, forcing the CMGs to track the input momentum profile (in an actual spacecraft implementation, this could be replaced by something like a PID controller to maintain commanded attitude rather than commanded momentum). Search decisions are made after every four integration steps (thus any trajectory is 15 search nodes deep). All of the above search parameters and CMG definitions can be readily varied, as discussed in [9].

The examples shown here allow only 3 children per parent node (i.e. negative null motion, no null motion, or positive null motion). Although the test software is able to employ an arbitrary branching density, this "ternary divert" strategy was seen to perform adequately, and minimizes the search complexity and execution requirement. The null motion scaling ( $\eta$ in Fig. 3b) was chosen such that at least one CMG was driven at $70 \%$ of its peak gimbal rate in nodes with null motion added. Referring to Eq. 12 , the objective weights used in this study were $\mathrm{W}_{1}=20, \mathrm{~W}_{2}=3, \mathrm{~W}_{3}=$ $2, \mathrm{~W}_{4}=100(\mathrm{sec} / \mathrm{rad})$, and $\mathrm{W}_{5}=0.05$. The maximum gimbal rate limit of $1 \mathrm{radian} / \mathrm{sec}$ was not violated in these examples; the heavy over-rate penalization in the objective and the robust SR-inverse calculation were seen to keep the gimbal rates well within bounds.

Execution requirements seem encouraging for application on future spacecraft. The simulations showed promising results when limiting the number of expanded nodes to under 6000 (2000 search steps, each expanding 3 child nodes per parent). Assuming that a data structure of roughly 20 bytes per node is adequate for managing the search and calculating the objective with 
sufficient accuracy[9], the search operation may be performed within 120 Kbytes (which can be released to other applications after the search concludes). On a Macintosh II computer (68020/68881 processor) running extremely inefficient, diagnostically-oriented, user-friendly software, the search was seen to usually approach convergence to its best solution within 1-2 minutes. A large gain in speed can easily be realized through tighter coding of the search algorithm. The search can be additionally hastened through application of parallel, dedicated processors, such as are now being proposed for eventual use in space-based robotics[18], resulting in execution times of seconds or less, and enabling near real-time operation.

The first example employs a constant torque command about the $\hat{x}$ axis. Such cases have been studied previously[7,12,16] for the 4-SGCMG pyramid. Although some tangent steering laws manage to satisfy this command, an unescapable singular state, which lurks at a net CMG momentum of $h_{x}=1.15$, is known to cause difficulty.

Search results are shown in Fig. 7, which depicts how the "currently best" trajectory adopted by the search evolves as the search progresses. The contour plots at left summarize the operation and performance of the search at each step. The overlaid curve follows the chosen nodeto-node trajectory (center is no null motion, top is negative null, bottom is positive null). The trajectory starts at the initial CMG state at the left of the plot, and achieves the final commanded momentum state at the right edge. The shading indicates the quality of the CMG state that is derived by varying the amount of null motion added to the SR inverse solution (i.e. sweeping the value of $R$ in Eq. 5 between its positive and negative limits). The darker the shade, the better the CMG state (i.e. the darkness of the shading is proportional to the CMG gain $m$ ). The vertical axis scaling is fixed at each timestep to span the maximum amount of null motion that can be currently added (recall that the null contribution $R$ is normalized at each node, such that the fastest gimbal is driven to within $\eta=70 \%$ of its peak rate).

The middle plots show the CMG gain $\mathrm{m}$ across the trajectory (horizontal axis is in commanded timesteps). The maximum vertical scale of these plots is fixed at the largest value of $m$ 
attainable with this CMG system $(m<2.4)$. The rightmost plots show the corresponding $\mathrm{CMG}$ gimbal angles, $\theta_{\iota}$.

Each row of plots corresponds to a sequentially chosen "best trajectory". The top row (a) is the initial trial consisting of the SR inverse only, with no added null motion. Thirty nodes were expanded at this trial, and an objective evaluation (Eq. 12) of -14.2 was achieved. The gimbal angle plot indicates that the commanded $\hat{x}$ torque was attained by "scissoring" two opposing gimbals, while leaving the other two nearly unaltered. The performance was quite poor, as seen from the plot of the CMG gain; a hard singular condition was held from time step 22 onward (this is the unescapable singularity at $\left.h_{x}=1.15\right)$. Since the singular direction is aligned with the commanded torque, the SR inverse produces no gimbal rates after the singularity is achieved, and sizeable momentum errors are accumulated.

The next accepted trajectory (b) is an early result from the search. The performance is considerably better, excepting a quick brush with the singular state at step 22 . The search thereupon rapidly improves the solution in steps (c-e). The dip in the CMG gain that indicated the approach of the singular state is quickly removed, and the average CMG gain is considerably increased, producing a highly controllable gimbal state. The last two trajectories $(\mathrm{d}, \mathrm{e})$ demonstrate the operation of the null motion objective component. Since the CMG gain is high throughout both of these trajectories, the null objective contribution becomes significant. Frequent changes in null motion are disfavored. The final solution (e), after 456 node expansions, was able to avoid the singularity with a single null motion pulse, achieving an objective evaluation of +21.0 , and resulting in a very satisfactory gimbal trajectory.

This example drove the CMG system only to within $50 \%$ of its momentum capacity. Searches that are run all the way out to the momentum envelope along the $+\hat{\mathrm{x}}$ axis are seen to find a solution similar to this one for the first half trajectory, and thereafter apply only the SR-inverse without null motion to reach the envelope (an early pulse of null motion, as shown in this example, was able to attain a superior gimbal state and avoid future singular conditions). 
Another example is given in Fig. 8. Here, the CMGs respond to equal torques simultaneously commanded about the $+\hat{x}$ and $-\hat{y}$ axes ( $\hat{z}$ torque remains zero). A set of singular states that are extremely difficult to avoid are known to be encountered through this command[7].

The initial trial with no null motion (a) produces the commanded $\hat{y}$ torque by scissoring all 4 gimbals. As seen in the $\mathrm{CMG}$ gain plot, a singularity is indeed achieved at time step 18. Because the singular direction is not aligned with the torque command in this case, the system is able to transit through the singularity and complete the momentum command. Nonetheless, the singular region is potentially unstable, and the objective encourages this encounter to be removed or minimized. The next accepted attempt (b) is the trial which tries to locally "unkink" the rotor projections through the method of Ref. [15]. Here, an initial shot of null motion pulls the system past a singularity at the start of the trajectory, after which a very high CMG gain is achieved. The following solution (c) is located by the search, and uses excessively changing null motion to further reduce the time spent near the singular state and keep $m$ large. This is additionally improved on; the final solution (d) specifies a gimbal trajectory (a variant on the unkinking technique) that spends only a very brief period near a singular configuration, with minimal null pulsing added. In this example, the singular state appears to be nearly unavoidable with the constraints (i.e. peak gimbal rates) imposed on the $\mathrm{CMG}$ system. The search did its best, however, to minimize both the approach to the singularity and the period of time spent at low CMG gain, as dictated by the objective of Eq. 12.

The sensitivities of these and other CMG trajectories to unmodelled disturbance torques have been examined in Ref. [9]. The feedforward null motion command $(R(t)$ determined by the search) was used by a local CMG steering law, as shown in Fig. 5. Gimbal trajectories that were able to avoid approach to a singular state (i.e. Fig. 7) were seen to be quite robust to added secular torques; predictability and performance were maintained with unmodelled steady-state torques exceeding $25 \%$ of the original torque commands used in the search. An example of this is shown in Fig. 9a, where the CMG gain from the trajectory of Fig. 7e is overlaid with the CMG gain produced after the addition of a constant unmodelled torque disturbance. This torque was 
distributed equally along all three axes (a worst-case direction for this example), and produced a $35 \%$ difference in the final net CMG momentum. A change in system performance can definitely be noted after the null motion pulse is applied (the perturbed trajectory yields lower gain), but the CMG system remains highly controllable. In this example, the perturbed gimbal angles were seen to diverge linearly[9] from the search results as the amount of injected disturbance increased; no abrupt change of trajectory was produced.

A different situation, however, was seen in cases with gimbal trajectories transiting a singularity (i.e. Fig. 8). The system was seen to reasonably track the feedforward gimbal reference until the singularity was approached. From this point onward, however, the steering law produced gimbal angles that could diverge considerably from the prediction of the search, even with only modest torque errors added. This is illustrated in Fig. $9 \mathrm{~b}$ for the trajectory of Fig. 8d. Although the constant disturbance torque (now only along the $\hat{x}$ axis) was comparatively smaller (causing a $15 \%$ shift in final CMG momentum), a totally different (and much less controllable) system performance was achieved after the singularity.

This analysis indicates that it is very difficult to predict past a singular state; in the singular region, many $\mathrm{CMG}$ closures[9] or gimbal trajectory possibilities patch together, thus a small change in the CMG condition (brought on by answering the unmodelled torques) can select a different gimbal configuration, yielding a rapid divergence, as characteristic of the chaotic dynamics seen in manipulator systems[19]. This may be interpreted physically. In a singular state, the rotor axes are all aligned to project maximally or minimally along a common axis, hence small perturbations will cause the rotors to "scissor" one way or another, producing very different subsequent gimbal configurations. A similar, but less severe, effect is seen[9] with gimbal trajectories that bring several $3 \times 3$ minors of the CMG Jacobian (Eq. 3) simultaneously near zero. Here various triplets of rotors lose rank (although the full CMG system remains nonsingular), and small perturbations can also produce "scissoring" in either direction and result in an abrupt change in the gimbal trajectory.

Although the divergence in the neighborhood of a singular state can be problematic for gimbal solutions that attempt to transit a singularity, several possibilities exist for recovery. One 
factor is a considerable aid; the locations of probable rapid divergence are known ahead of time to be at the gimbal states where the search had indicated proximity to a singularity. This allows some contingencies to be taken; i.e. the gimbals can be constrained to follow a particular path when traversing the singular state such that the gimbal state is pre-known both before and after the singularity is crossed. The results of two feedforward searches can then be applied; one leading up to the singular state, and another from the opposite side of the singularity onward. Other techniques of recovering from gimbal divergence are possible, as suggested in Ref. [9].

The search implementation used in these examples allowed only three levels of null motion; i.e. negative, zero, or positive. Although gimbal chatter was minimized through the objective function, greater dynamic range and smoother gimbal response may be achieved by generating a larger number of children per parent node, or allowing the search to vary the change in null motion at each step, rather than its net value.

The CMG gimbal trajectory can be easily be coded as a "character" string, with each character representing the added null motion, and its position in the string specifying the corresponding momentum step. The optimality of these trajectory strings can be described by the cost of the trailing character (i.e. terminal node), as calculated through Eq. 7. A genetic algorithm[20] is a powerful method of efficiently locating families of such strings (i.e. "populations") representing groups of related, near-optimal solutions. This technique may provide a superior means of globally specifying desirable CMG gimbal trajectories in future efforts.

\section{Conclusions}

A global search has been shown to be effective in producing singularity-avoiding feedforward CMG gimbal trajectories in response to a command history forecast from a momentum management or maneuver scheduler. In cases where the singularity is unavoidable, the 
search acts to minimize the severity of the singular encounter (i.e. transits the singularity as rapidly as possible, with minimum induced momentum error). Gimbal trajectories that are removed from singular encounters have been noted to be appreciably robust to the introduction of unmodelled torque error. Trajectories that skirt a singular state, however, can show significant sensitivity and quickly diverge from the feedforward reference after the singular approach. Since the location of probable divergence is thus known a priori, one may apply constraints near the singular region that can maintain the anticipated system performance.

\section{Acknowledgements}

John Sunkel and Lynda Bishop of NASA/JSC are earnestly thanked for their interest and support in this effort. The information on search techniques provided by Owen Deutsch and Bob Beaton of CSDL is greatly appreciated. The many spirited CMG discussions held with Nazareth Bedrossian (CSDL/MIT) and Haruhisa Kurokawa (MEL, Japan) were invaluable. This work was supported under NASA contract NAS9-18147. This paper is published solely for the exchange and stimulation of ideas, and does not constitute approval by NASA of the findings or conclusions contained herein.

\section{References}

1) Kennel, H.F., "Steering Law for Parallel Mounted Double-Gimballed Control Moment Gyros Revision A," NASA TM-82390, Jan. 1981.

2) Yoshikawa, T., "A Steering Law for Three Double-Gimballed Control Moment Gyro Systems," NASA TM X-64926, March 1975.

3) Paradiso, J.A., "A Highly Adaptable Steering/Selection Procedure for Combined CMG/RCS Spacecraft Control," 1986 AAS Guidance and Control Conf., Keystone, CO., AAS 86-036, Adv. Ast. Sci. Vol. 61, pp. 263-280, Feb. 1986. 
4) Kennel, H.F., "A Control Law for Double-Gimballed Control Moment Gyros Used for Space Vehicle Attitude Control," NASA TM X-64536, Aug. 7, 1970.

5) Meffe, M., "Control Moment Gyroscope Configurations for the Space Station," 1988 AAS Guidance and Control Conf., Keystone, CO., AAS 88-040, Jan. 1988.

6) Branets, V.N. et. al., "Development Experience of the Attitude Control System Using SingleAxis Control Moment Gyros for Long-Term Orbiting Space Stations," Proc. of the 38'th Congress of the International Astronautical Federation, Brighton, UK, Oct. 1987.

7) Bedrossian, N., Paradiso, J., Bergmann, E., Rowell D., "Steering Law designs for Redundant SGCMG Systems," AIAA Journ. of Guidance \& Control, Vol. 13, No. 6, pp. 1083-1089, Nov.Dec., 1991.

8) Margulies, G., Aubrun, J.N., "Geometric Theory of Single-Gimbal Control Moment Gyro Systems," Journ. Ast. Sci., Vol. 26, No. 2, April/June 1978, p. 159.

9) Paradiso, J.A., "A Search-Based Approach to Steering Single Gimballed CMGs," C.S. Draper Laboratory report CSDL-R-2261, August, 1991.

10) Kurokawa, H., "Topological Study of Single Gimbal Control Moment Gyro Systems," to be published.

11) Kurokawa, H., Yajima, N., Usui, S., "A New Steering Law of a Single-Gimbal CMG System of Pyramid Configuration," Proc. of the X'th IFAC Symposium on Automatic Control in Space, Toulouse, France, 25-28 June, 1985, p. 249.

12) Bauer, S.R., "Single Gimbal CMG Steering Laws", C.S. Draper Lab. Report SGN Memo 10E87-06, May 1987. 
13) Bedrossian, N., Paradiso, J., Bergmann, E., Rowell, D. "Redundant Single Gimbal CMG Singularity Analysis," AIAA Journ. of Guidance \& Control, Vol. 13, No. 6, pp. 1096-1101, Nov.Dec., 1991.

14) Strang, G., Introduction to Applied Mathematics, Wellesley-Cambridge Press, Wellesley, MA., 1986.

15) Cornick, D.E., "Singularity Avoidance Control Laws for Single Gimbal Control Moment Gyros," 1979 AIAA Guidance and Control Conf., Boulder, CO., AIAA 79-1968, pp. 20-23, Aug. 1979.

16) Vadali, S.R., Oh, H-S, Walker, S.R., "Preferred Gimbal Angles for Single Gimbal Control Moment Gyros," AIAA Journ. of Guidance \& Control, Vol. 13, No. 6, pp. 1090-1095, Nov.-Dec., 1991.

17) Tokvar, E.N. and Platanov,V.N., "Singular Surfaces in Unsupported Gyrodine Systems," Cosmic Research, Vol. 16, No. 5, 1978, pp. 547-555.

18) Fijany, A., Bejczy, A., "Parallel Architecture for Robotics Computation," NASA/JPL Invention Report NPO-17629/7126, June 1990.

19) Lokshin, A., Zak, M.A., "Chaotic Motion of a Two-Link Planar Mechanism," NASA/JPL Invention Report NPO-17387/6898, Aug. 1989.

20) Goldberg, D.E., Genetic Algorithms in Search, Optimization, \& Machine Learning, AddisonWesley, 1989. 


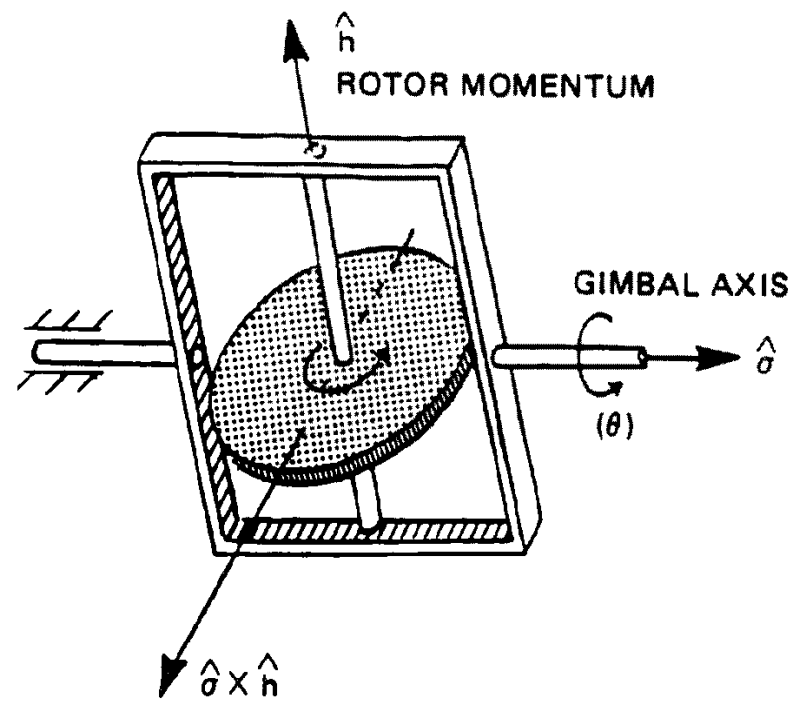

Figure 1: A Basic SGCMG 


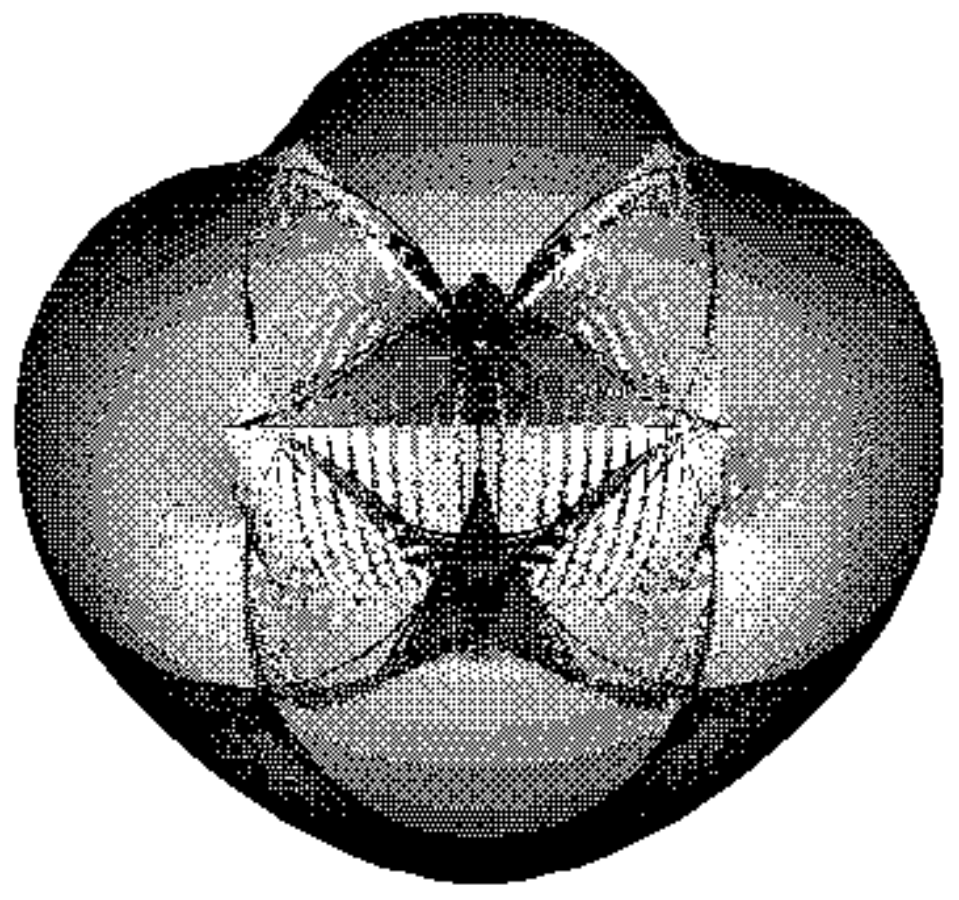

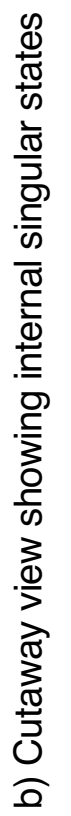

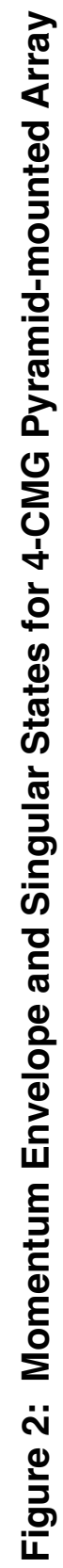




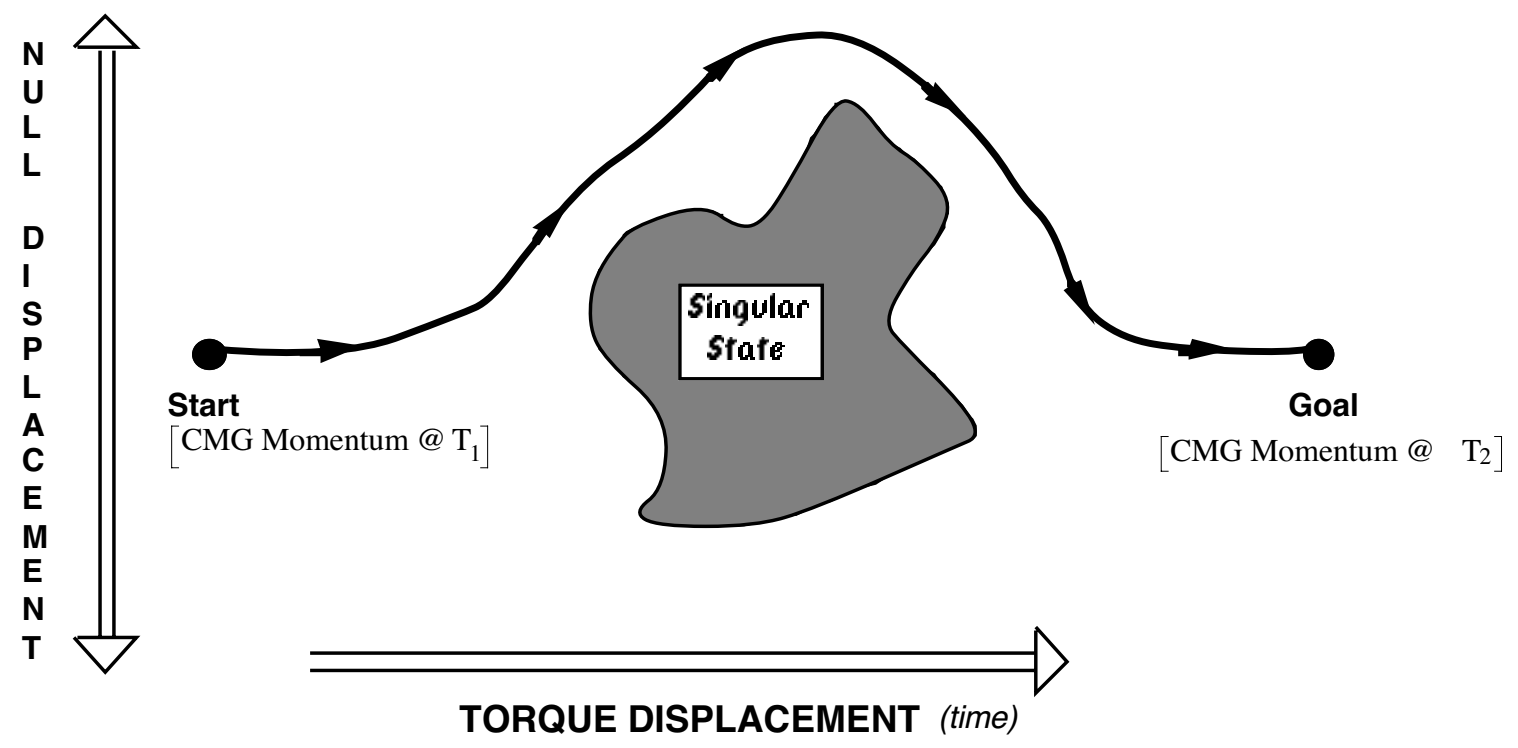

a) Coordinate Definition

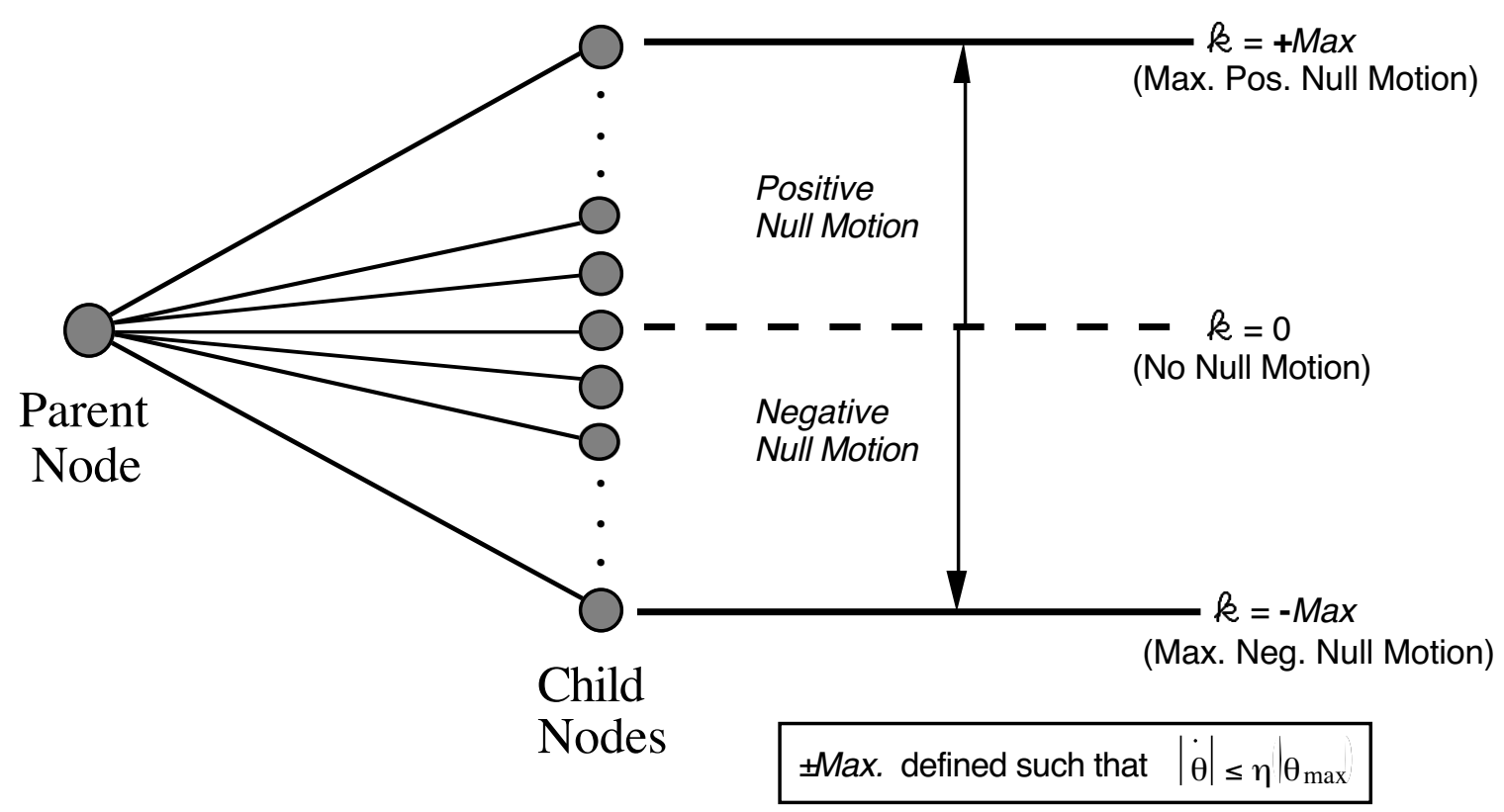

b) Node Structure

Figure 3: Coordinate and Node Definitions for CMG Search 


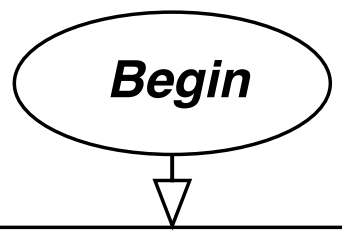

Run selected gradient strategies for "trial" trajectories to initialize costs \& generate "experience". All nodes are made "OPEN" for expansion by the subsequent search. Cost cutoff is set to the best completion cost found in the trials. The "best trajectory" is assigned to the best-cost trial.

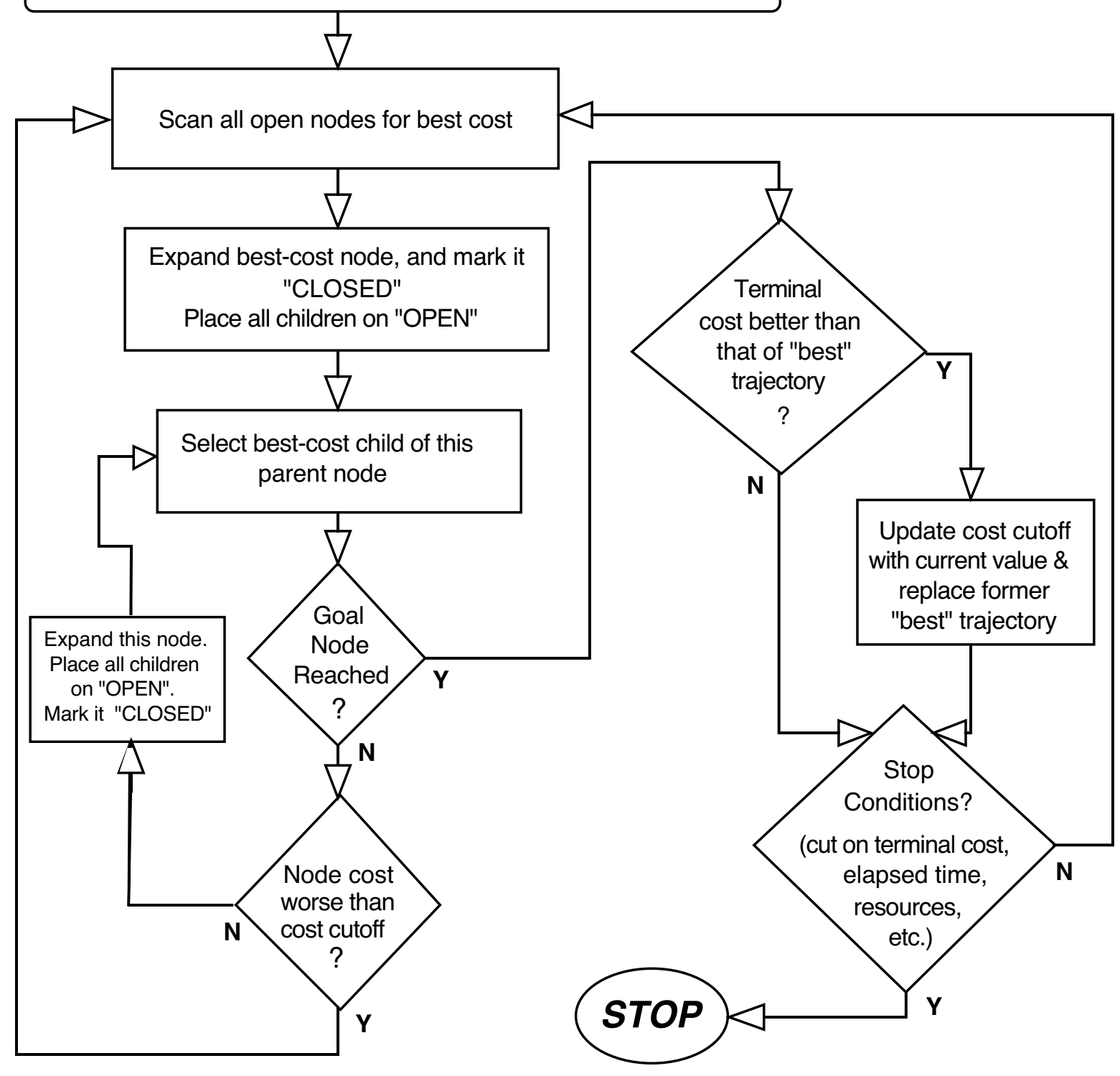

Figure 4: Flow Diagram for Guided Depth-First CMG Search 


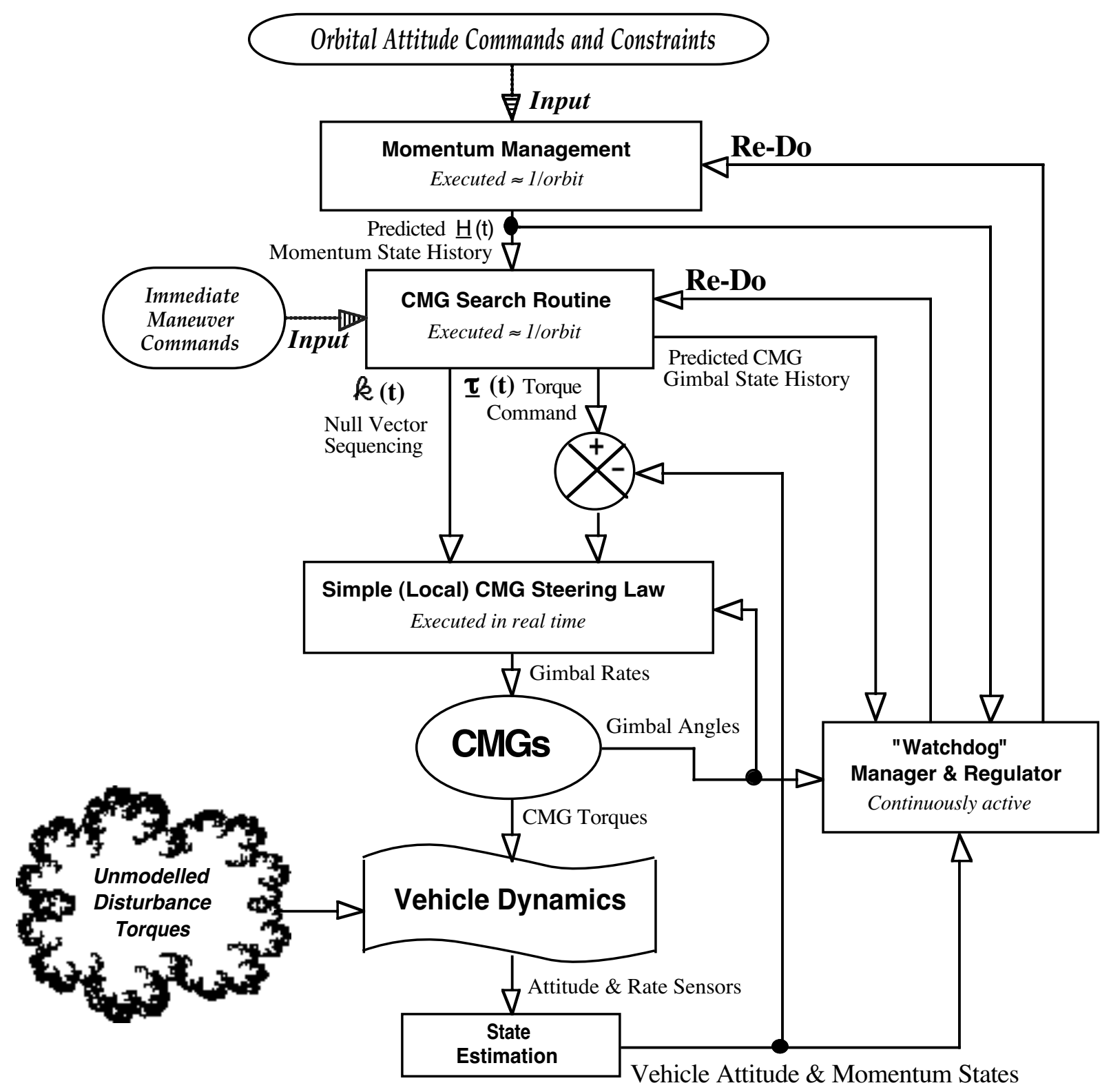

Figure 5: Logic for Orbital Implementation of Search-Based CMG Steering 


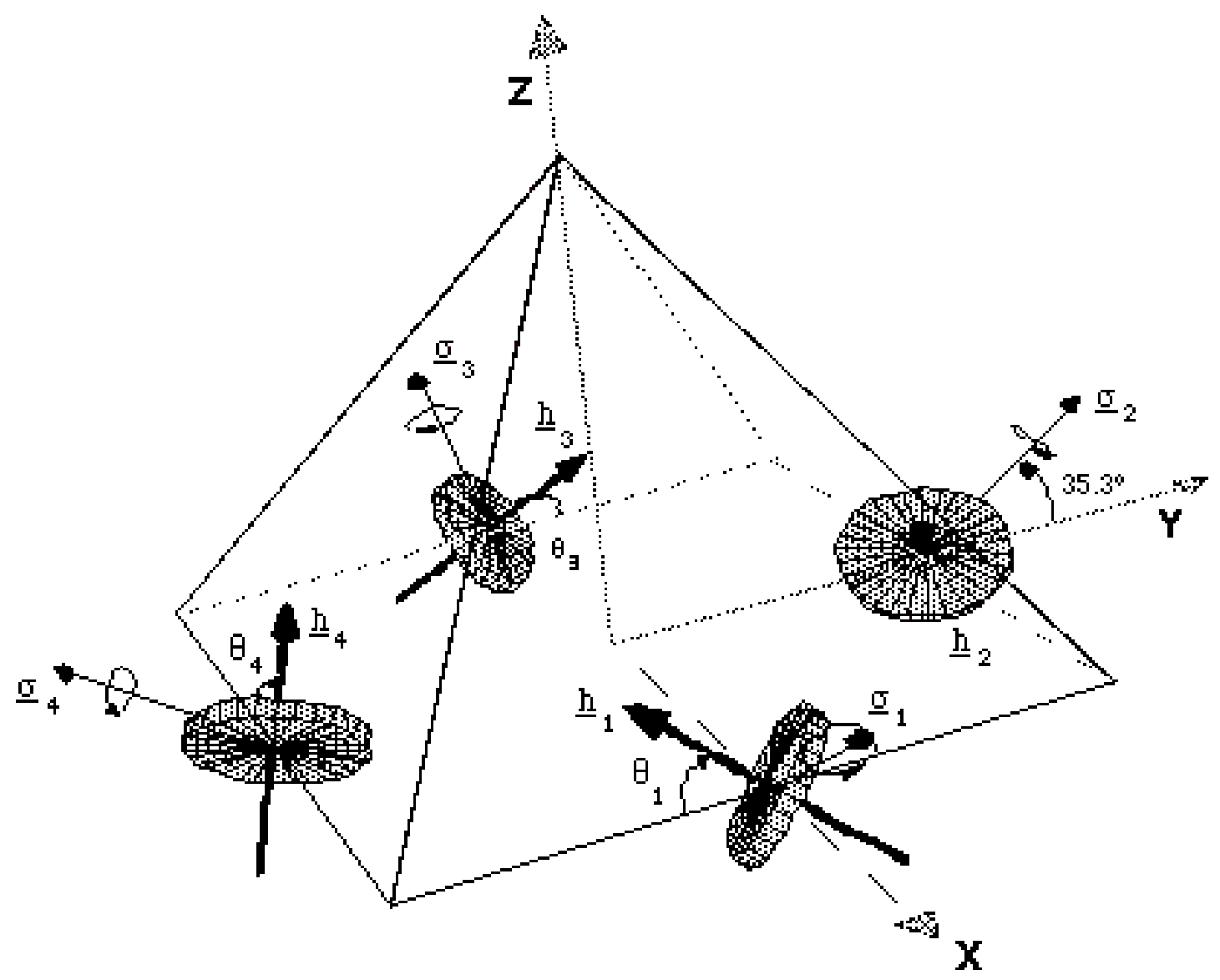

Figure 6: 4-CMG Pyramid Mounting Scheme 


\section{Search Performance}
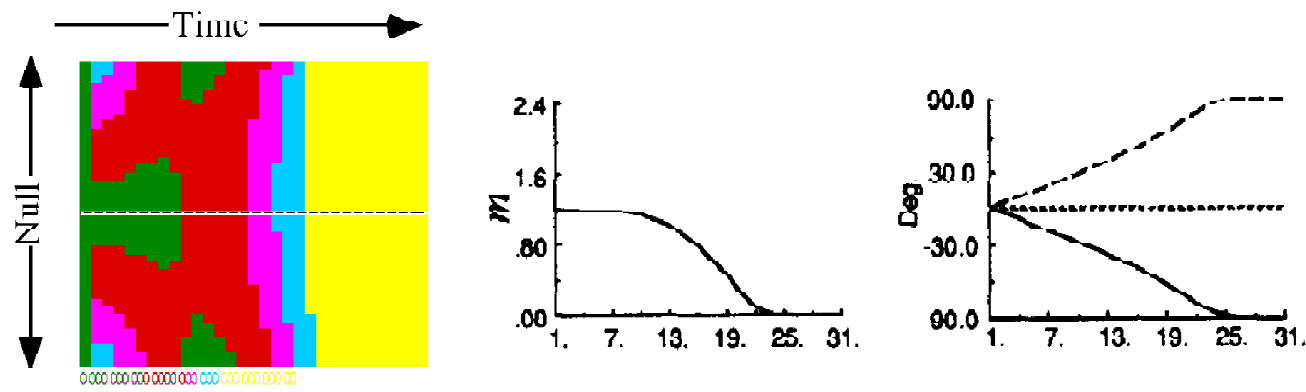

a) No Null Motion: 30 Expanded Nodes. Objective $=-14.2$
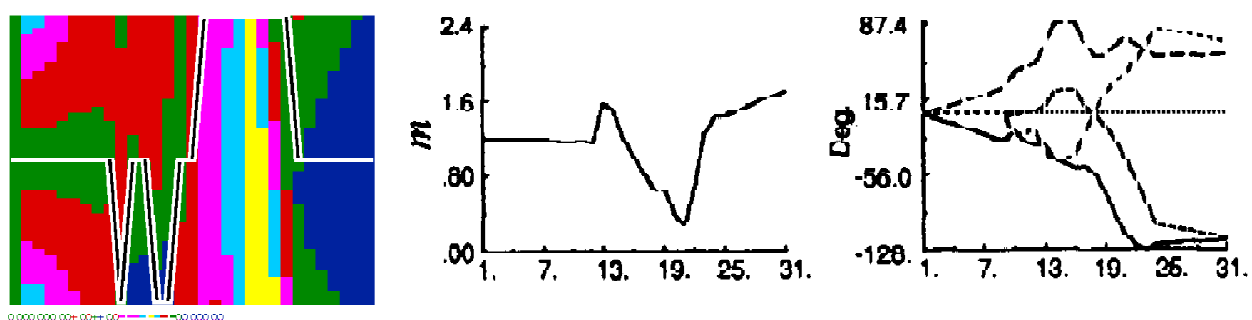

b) Search Result; 164 Expanded Nodes, Objective $=+3.32$
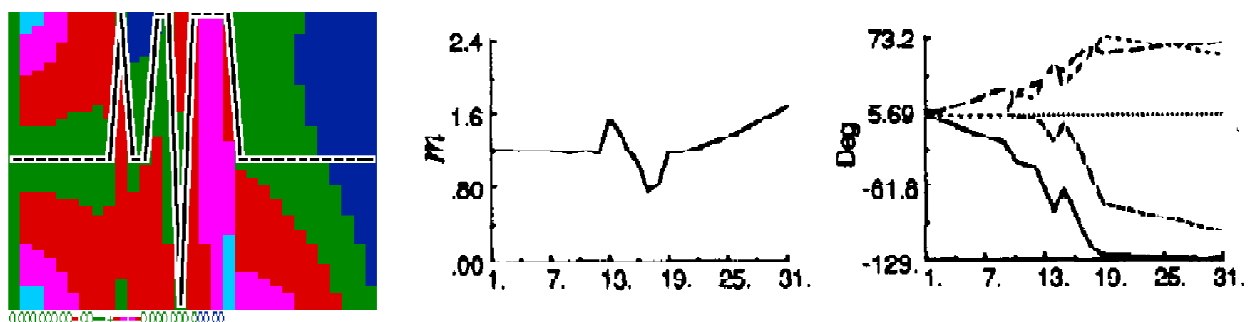

c) Search Result: 279 Expanded Nodes, Objective $=+13.3$
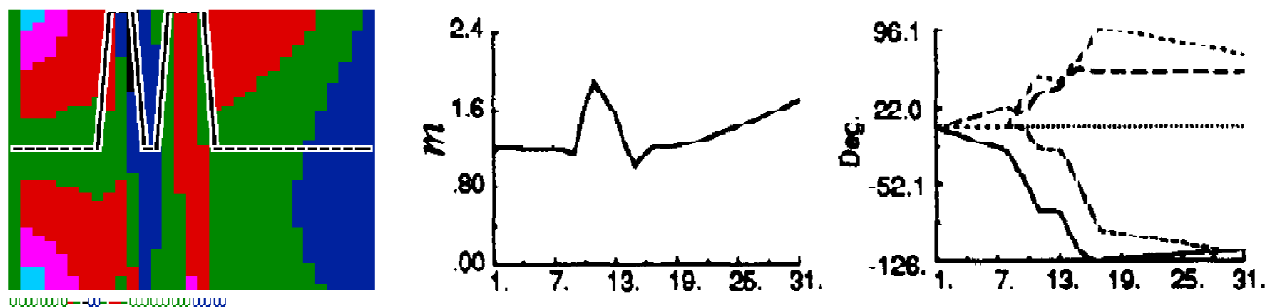

d) Search Result; 355 Expanded Nodes, Objective $=+19.2$
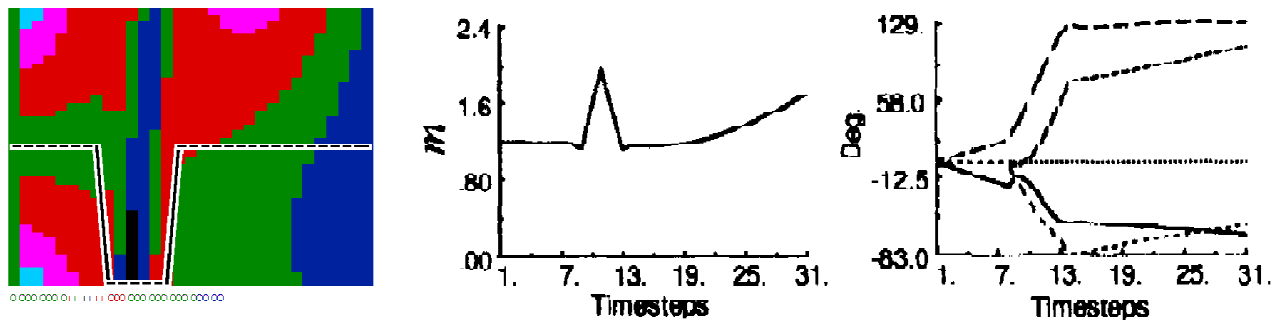

c) Scarch Hosult; 456 Expandcd Nodcs, UbjcctIvc $=+21.0$

Figure 7: CMG Search Performance for Commanded Torque About $+\hat{\mathbf{x}}$ Axis 
Search Performance

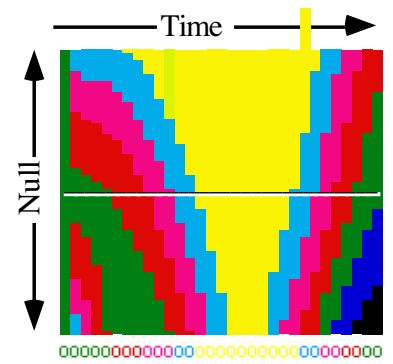

CMG Gain

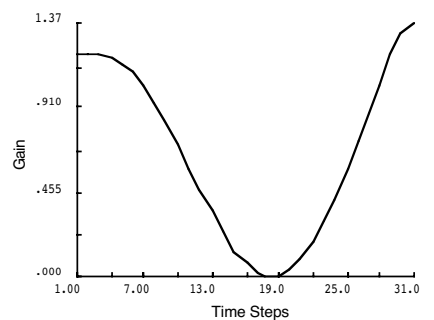

Gimbal Angles

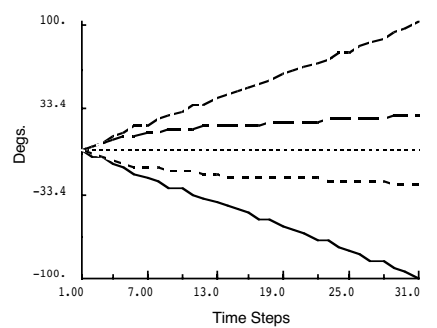

a) No Null Motion; 30 Expanded Nodes, Objective $=-8.57$
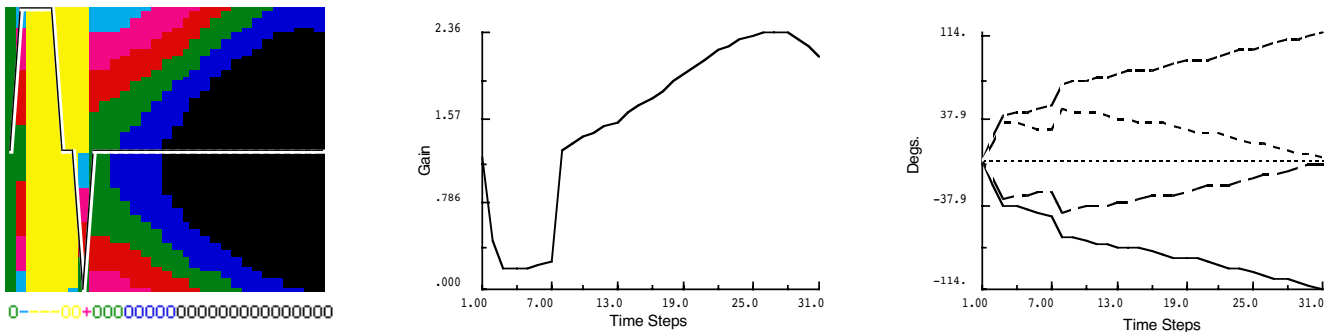

b) "Unkinking" Trial; 144 Expanded Nodes, Objective $=-2.03$
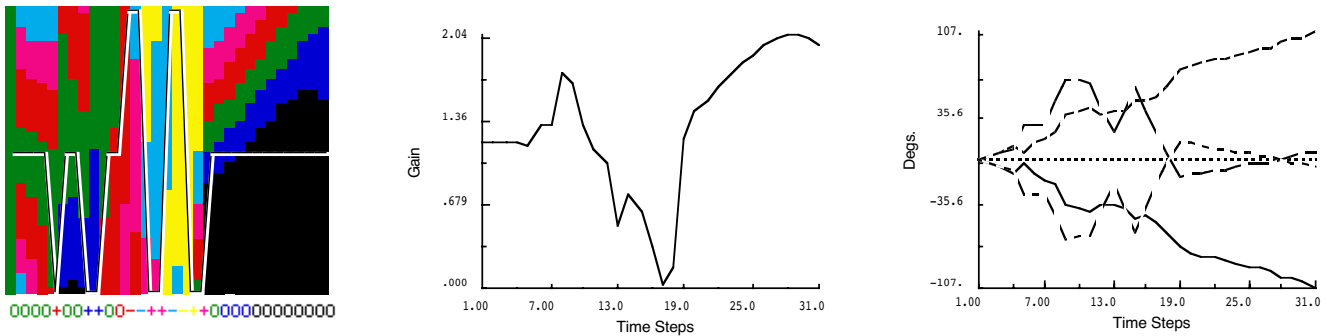

c) Search Result; 219 Expanded Nodes, Objective $=-1.69$
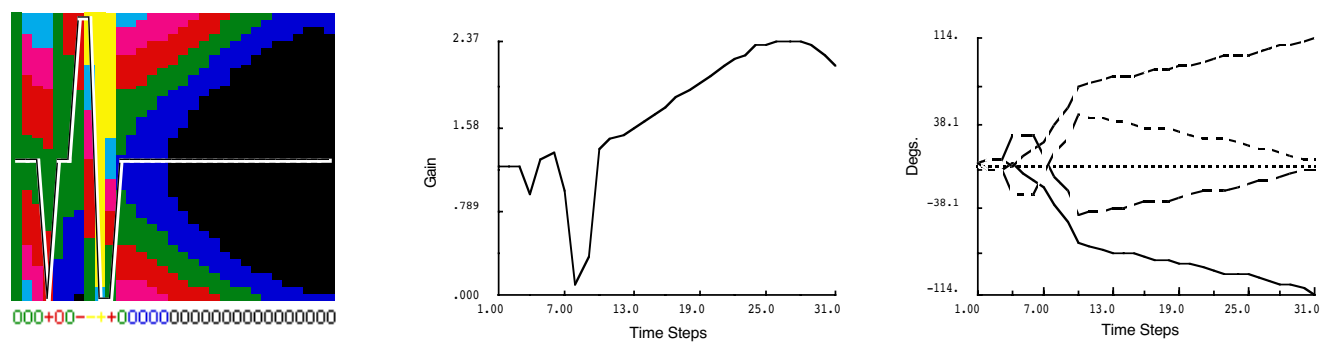

d) Search Result; 428 Expanded Nodes, Objective $=+0.19$

Figure 8: CMG Search Performance for Commanded Torques About $+\hat{x}$ and $-\hat{y}$ Axes 


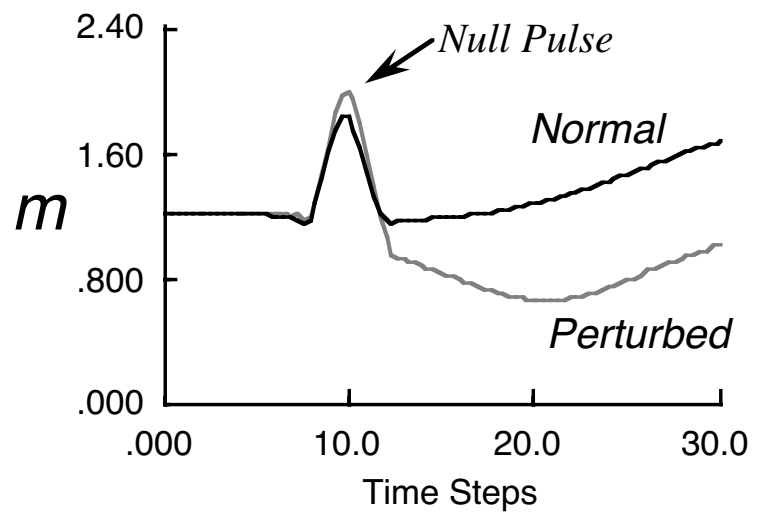

a) Constant Torque About $+\widehat{\mathbf{x}}$ Axis

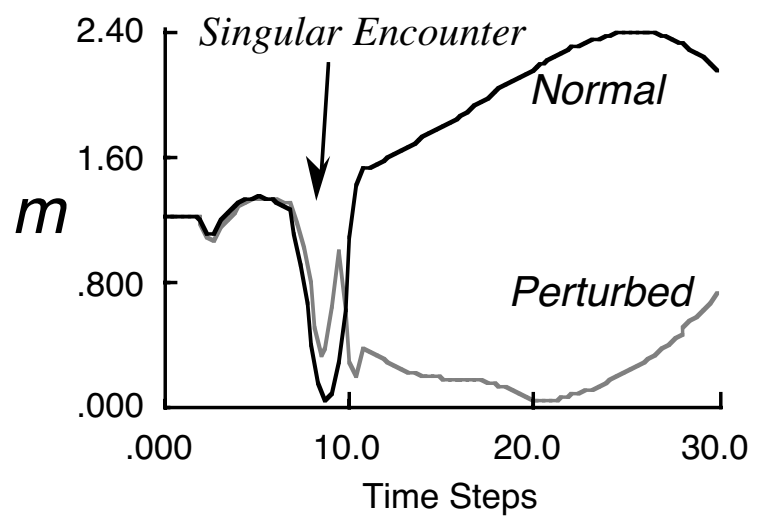

b) Constant Torque About $+\widehat{\mathbf{x}},-\widehat{\mathbf{y}}$ Axes

Figure 9: Response to Unmodelled Bias Torques 


\section{Figure Captions}

Figure 1: A Basic SGCMG

Figure 2: Momentum Envelope and Singular States for 4-SGCMG Pyramid-mounted Array

Figure 3: Coordinate and Node Definitions for CMG Search

Figure 4: Flow Diagram for Guided Depth-First CMG Search

Figure 5: Logic for Orbital Implementation of Search-Based CMG Steering

Figure 6: 4-CMG Pyramid Mounting Scheme

Figure 7: CMG Search Performance for Commanded Torque About $+\hat{\mathrm{x}}$ Axis

Figure 8: CMG Search Performance for Commanded Torques About $+\hat{x}$ and $-\hat{y}$ Axes

Figure 9: Response to Unmodelled Bias Torques 